\title{
Advancements in automated drop setting and imaging for high throughput crystallization
}

\author{
Mayank Aggarwal, Ph.D. - Formulatrix \\ lance.ramsey@formulatrix.com
}

Over the past 10 years, automation for crystallization has been widely adopted and has become general practice in most industrial and academic labs. The use of automation has increased throughput and resulted in an explosion of structure determinations. As automation has become the norm, creativity from scientists has directed implementation of new hardware solutions and continual improvement. Recently, Formulatrix has optimized the use of their drop setter, the $N T 8 \circledast$, to perform seeding experiments which has been long known to provide an initial nucleation point and thus increase the number and quality of crystals that grow in a particular condition. Reliable drop setting is the backbone of crystallization experiments and the NT8 simplifies this often tedious step while increasing reproducibility with tight humidity control. Imaging is also essential in finding positive crystallization hits with Formulatrix offering a suite of different options. Recently, visible fluorescence imaging options have been brought to market allowing scientists to accurately distinguish between single protein crystals and protein-protein complex co-crystals, as well as detect protein crystals with ultra-high sensitivity and shorter imaging times. Nonlinear imaging, SONICC, has proven to be exceptional for detecting submicron crystals and identifying positive crystallization conditions never seen before. Recent advancements in imaging methods and detectors has led to a vast improvement in signal to noise allowing identification of even more crystals. Software solutions to aid in automatic scoring of drops has also recently been implemented. We have opened up our Rock Maker software to allow the coupling of machine learning algorithms to work within Rock Maker and automatically score drops without human intervention. With each year comes new ideas and solutions to solve our biggest challenges in structure determination, with Formulatrix striving to meet the needs of the crystallographers. 